Electrochemical Study of Hydrogen Permeation through

Tungsten near Room Temperature

\author{
A. Manhard", S. Kapser, L.Gao \\ Max-Planck-Institut für Plasmaphysik, Boltzmannstr. 2, D-85748 Garching, Germany
}

NOTICE: This is the author's version of a work that was accepted for publication in Journal of Nuclear Materials. Changes resulting from the publishing process, such as peer review, editing, corrections, structural formatting, and other quality control mechanisms may not be reflected in this document. Changes may have been made to this work since it was submitted for publication. A definitive version was subsequently published in

J. Nucl. Mater. 463, (2015) pp. 1057-1061, DOI:10.1016/j.jnucmat.2014.10.018 


\title{
Electrochemical Study of Hydrogen Permeation through Tungsten near Room Temperature
}

\author{
A. Manhard*, S. Kapser, L.Gao \\ Max-Planck-Institut für Plasmaphysik, Boltzmannstr. 2, D-85748 Garching, Germany
}

\begin{abstract}
We used an electrochemical double cell to study permeation of hydrogen through a $3.5 \mu \mathrm{m}$ thick sputter-deposited tungsten layer on a $25 \mu \mathrm{m}$ thick palladium support. The temperature dependence of the steady-state permeation current was studied in the range from 266 to $333 \mathrm{~K}$ for a constant charging potential on the entry side and zero hydrogen concentration on the exit side of the sample foil. We found that the data is best described by a sum of two Arrhenius terms. We postulate that the higher activation energy of $0.86 \pm 0.07 \mathrm{eV}$ corresponds to permeation through bulk grains and may approach the literature value of $1.43 \pm 0.26 \mathrm{eV}$ for even higher temperature. For the second, lower activation energy of $0.39 \pm 0.03 \mathrm{eV}$, we currently consider fast diffusion along grain boundaries the most likely explanation.
\end{abstract}

PSI-21 Keywords: Permeation, Hydrogen, Tungsten, Coating

PACS: 66.30.J- (diffusion of impurities), 82.45.-h (electrochemistry), 81.15.Cd (deposition by sputtering)

*Corresponding Author: Armin Manhard

Presenting Author: Armin Manhard

E-mail: armin.manhard@ipp.mpg.de

Adress: Max-Planck-Institut für Plasmaphysik, Boltzmannstr. 2, D-85748 Garching, Germany 


\section{Introduction}

Permeation experiments are a common way to study hydrogen transport in metals (see [1] and references therein). Typically, such experiments are performed by exposing one surface of a metal membrane to either a hydrogen atmosphere, or alternatively to a $\mathrm{H}$ isotope ion beam respectively plasma, and measuring the permeation flux into an ultra-high vacuum chamber on the other side of the membrane. Performing such experiments at elevated temperatures serves to increase the permeation flux and reduce the permeation time lag. For tungsten, there is only little data available [2], particularly for temperatures below $500 \mathrm{~K}$. To describe processes involving $\mathrm{H}$ isotope diffusion in $\mathrm{W}$ in this temperature range, one way is to extrapolate from data acquired at higher temperatures, such as those measured by Frauenfelder [3], which are currently most generally accepted [2, 4]. Alternatively, the diffusion parameters are derived from numerical models describing experiments performed in this temperature range with $\mathrm{H}$ ion beams or plasmas (see, e.g., [5]). Both suffer from considerable uncertainties. A notable exception is the gas-driven permeation of tritium through tungsten foils, which has been recently performed by Ikeda et al. [6]. However, the use of radioactive tritium gas requires extensive safety measures and can therefore only be performed in highly specialised laboratories. For the accurate modelling of $\mathrm{H}$ isotope implantation experiments, which are often performed in this temperature range (see, e.g., $[2,4]$ and references therein), it is necessary to provide a broader, more precise data base on $\mathrm{H}$ isotope diffusion in $\mathrm{W}$. Such a data base will also be very helpful for accurately predicting H isotope losses during storage of samples (see, e.g., [7]) - or even entire plasma-facing components from fusion experiments - after their exposure to plasma. Finally, permeation experiments at low temperatures are helpful for studying the low-energy part of the binding energy spectrum of $\mathrm{H}$ isotopes to defects in tungsten. 
We present permeation experiments using an electrochemical double cell as an alternative, highly sensitive method for permeation studies near room temperature [8]. This method has already been successfully applied to a number of metal-hydrogen systems, such as $\mathrm{H}$ in palladium or in iron (see, e.g., $[8,9,10])$. The basic principle is described, e.g., in [11] and is shortly summarised here: Hydrogen atoms are supplied from a proton-containing electrolyte in the charging cell on one surface of the metal sample by applying a cathodic potential. These hydrogen atoms are dissolved in the metal and diffuse to the other side of the sample, where they are ionised by a sufficiently high anodic potential and dissolved again in the electrolyte of the recipient cell. The resulting current on the anodically polarised side of the sample is a direct measure for the permeating hydrogen flux. Because there is no pressure difference between both cells, very thin membranes can be used. This reduces the diffusion timescale and increases the permeation flux. In this article we demonstrate for the first time the successful application of an electrochemical permeation experiment to protium $\left({ }^{1} \mathrm{H}\right)$ in tungsten.

\section{Experiment description}

\subsection{Electrochemical double cell}

The basic set-up of the experiment is a galvanic double cell as described by Devanathan and Stachurski [8] (Figure 1). It consists of two double-jacketed glass vessels separated by the sample membrane. The counter electrodes are made of platinum mesh, the reference electrodes are saturated calomel electrodes (SCE). The electrolyte is a highly viscous mixture of two volume parts glycerol and one volume part concentrated phosphoric acid (85\%), as suggested by Kirchheim and McLellan [9]. In this electrolyte, phosphoric acid supplies the protons, while glycerol mainly acts as a filler to increase the viscosity of the mixture.

Compared to other, more commonly used electrolytes such as dilute sulphuric acid or aqueous sodium hydroxide solution, it has the advantage of a much lower background current. This 
current is believed to arise from the reduction of gas molecules dissolved in the electrolyte and by formation of $\mathrm{H}_{2}$ molecules at the sample surface (see, e.g., [9, 12]). The electrolyte was de-gassed in vacuum for at least 24 hours before filling it into the permeation set-up. Because the electrolyte is hygroscopic and water uptake from the ambient atmosphere would deteriorate its favourable properties [9], both cells are continuously purged with nitrogen at a minimal overpressure, which is monitored by an expansion balloon in one of the cells. The $\mathrm{N}_{2}$ lines for both cells are connected to equilibrate the gas pressure. Currents and voltages for both cells are supplied and measured by a BioLogic SP-300 two-channel potentiostat. The channels are electronically synchronised for the measurements presented here. A Pt-100 sensor inside a thin glass tube for corrosion protection monitors the electrolyte temperature. The tube is filled with a water-ethanol mixture for better thermal contact. We verified the accuracy of the temperature measurement by filling the cells with ethanol and comparing the readings from a sensor in the glass tube and a sensor immersed directly in the ethanol.

\subsection{Permeation sample}

The investigated sample was a $3.5 \pm 0.1 \mu \mathrm{m}$ thick PVD tungsten film deposited by magnetron sputtering onto a $25 \mu \mathrm{m}$ thick Pd foil. The deposition was performed in the same apparatus (Discovery ${ }^{\circledR} 18$, Denton) and by the same recipe as described by Wang et al. [13, 14], i.e., DC biasing of the sputter target with a power of $300 \mathrm{~W}$ at an argon gas pressure of $0.5 \mathrm{~Pa}$. The only difference was that pre-sputtering of the substrate foil was performed as gently as possible with our set-up with only about $-150 \mathrm{~V}$ self-bias for 30 minutes. Based on the analysis of Wang et al. of such PVD-W films [13], we assume that the impurity levels are all below 1 at.\% in our films. This analysis also suggests that the density of the $\mathrm{W}$ films is of the order of $90-95 \%$ of ideal bulk W $[13,14]$. After W deposition on the Pd foil, the free tungsten surface was cleaned for 30 minutes by a capacitively coupled Ar plasma with a selfbias of $-100 \mathrm{~V}$ and coated with $\sim 50 \mathrm{~nm}$ of Pd by electron beam evaporation. The Pd protects 
the $\mathrm{W}$ against corrosion in the electrolyte. It also facilitates a quick equilibration of the membrane surface with the hydrogen-containing electrolyte (see, e.g., [11]). I.e., cathodically supplied $\mathrm{H}$ is first absorbed by the Pd layer. Due to the high diffusivity and solubility of $\mathrm{H}$ in $\mathrm{Pd}$, the concentration of dissolved $\mathrm{H}$ in this very thin layer is approximately homogeneous and in equilibrium with the $\mathrm{H}^{+}$activity in the electrolyte at all times. The $\mathrm{H}$ concentration in $\mathrm{W}$ at the Pd-W interface then adjusts so that the respective activities of $\mathrm{H}$ in $\mathrm{Pd}$ and $\mathrm{W}$ become equal again. The anodic polarisation at the exit side enforces effectively zero $\mathrm{H}$ concentration in the Pd support foil, which leads also to zero $\mathrm{H}$ concentration on the exit side of the W layer. Because the solubility and the diffusivity of $\mathrm{H}$ in $\mathrm{Pd}$ (and therefore the permeability) are orders of magnitude larger than for $\mathrm{H}$ in $\mathrm{W}$ in the temperature range investigated here, the influence of the Pd layer as well as of the Pd support on the permeability is considered small according to considerations by Kumnick and Johnson [10]. After the permeation experiments were finished, we prepared a cross-section of the sample by focused ion beam (FIB). A scanning electron micrograph of this cross-section is shown in Figure 2. The grain size within the W layer is much smaller than that of the Pd substrate. Their size depends on the underlying Pd grain, as well as the elongation direction of the needle-like W grains. Quantitative analysis of the W microstructure has not yet been performed, but is planned.

\subsection{Measurement of the steady-state permeation current}

In this article, we present the investigation of the temperature dependence of the steady-state permeation current $j_{\infty}(T)$. For this, we fixed the electrochemical potential at the entry and exit sides of the sample (potentiostatic mode). The entry side was cathodically polarised to $E_{\text {charge }}=-350 \mathrm{mV}$ vs. SCE, the exit side was set to an anodic potential of $E_{\text {exit }}=+400 \mathrm{mV}$ vs. SCE. The anodic potential $E_{\text {exit }}$ is sufficiently high to keep the H concentration at the exit surface approximately at zero at all times, but not so high as to lead to a significant background current due to $\mathrm{H}_{2}$ formation at the Pt counter electrode. The current $j_{\infty}(T)$ was 
measured in the cell on the exit side and is a direct measure for the permeating $\mathrm{H}$ flux. The charging current $j_{\text {in }}(T)$ in the cell on the entry side was about 1.3 to 2 times higher than the permeation current in steady state, i.e., the transfer efficiency of $\mathrm{H}$ through our sample was of the order of $50-75 \%$. It followed a similar temperature dependence as $j_{\infty}(T)$. The excess hydrogen on the entry side that did not permeate through the sample was most likely lost either by $\mathrm{H}_{2}$ molecule formation at the sample surface, or by diffusion parallel to the sample surface within the thin Pd cover layer.

For a given temperature, the constant cathodic charging potential means a fixed, finite $\mathrm{H}$ concentration at the entry surface. At the exit side, the solute $\mathrm{H}$ concentration is fixed at effectively zero for all investigated temperatures, i.e., all permeating $\mathrm{H}$ is immediately ionised and transported away through the electrolyte of the recipient cell. To minimise the measurement noise, we used the built-in $5 \mathrm{~Hz}$ low-pass analog filter of the potentiostat and set the integration time to 10 seconds. Data were acquired continuously during the whole experiment.

Once stationary permeation conditions are reached, i.e., when the permeation current has become constant, $j(t)=j_{\infty} \approx$ const., the concentration gradient of interstitially dissolved hydrogen is also constant. In this state, $j_{\infty}$ is directly proportional to the $\mathrm{H}$ concentration at the entry side $c_{0}(T)$ and to the diffusion coefficient $D(T)$. The $\mathrm{H}$ concentration $c_{0}(T)$ is linked to the charging potential $E_{\text {charge }}$ through the solubility $S(T)$. As a result, $j_{\infty}(T)$ is proportional to the product of $S(T)$ and $D(T)$, which is called the permeability $\Phi(T) \propto \exp \left(-E_{\text {perm }} / k_{B} T\right)$. The activation energy $E_{\text {perm }}$ is therefore the sum of the activation energies for diffusion, $E_{\text {diff, }}$ and dissolution, $E_{\text {sol }}$, of $\mathrm{H}$ in W. An Arrhenius plot of $j_{\infty}(T)$ versus $1 / T$ yields this activation energy $E_{\text {perm. }}$. Please note that by measuring the steady state permeation current $j_{\infty}(T)$, it is not 
possible to distinguish the two individual contributions, $E_{\text {diff }}$ and $E_{\text {sol }}$, to $E_{\text {perm }}$.

The measurement of the steady-state current $j_{\infty}$ implicates that trapping and de-trapping of $\mathrm{H}$ must be in equilibrium and do not influence the solute $\mathrm{H}$. Accordingly, $j_{\infty}$ is a quantity that is independent of the traps in the material. The influence of trapping is only seen during nonstationary phases of the experiment. With our measurement, we therefore probe properties of interstitial $\mathrm{H}$ in $\mathrm{W}$ at temperatures where traps often dominate the overall behaviour.

Over a total period of about 8 days, the steady-state permeation current was measured for temperatures between 266 and $333 \mathrm{~K}$. The low-temperature limit was imposed to avoid freezing of the electrolyte. The high-temperature limit of $333 \mathrm{~K}$ was imposed to avoid damaging of the sample and parts of the permeation set-up by the hot, concentrated acid in the electrolyte.

\section{Results}

The raw data measured for $j_{\infty}(T)$ are indicated by the open squares in Figure 3 . The first data point was recorded at $280 \mathrm{~K}$. The following data points were acquired in random order alternating between high and low temperatures. To monitor the effects of sample and electrolyte ageing during the whole measurement period, we repeated the measurement at $280 \mathrm{~K}$ several times as a drift reference (see arrow in Figure 3). The raw data points in Figure 3 show some scatter, although the individual current measurements were highly accurate and nearly noise-free (RMS significantly smaller than $1 \mathrm{nA} / \mathrm{cm}^{2}$ in all cases). The error bars shown for the raw data in Figure 3 actually reflect the small, slow residual drift of $j_{\infty}(T)$ after changing the temperature, rather than measurement noise. The scatter of the raw data, which is much larger than even these error bars, is due to a long-term degradation of $j_{\infty}$ over the entire measurement period. We attribute this degradation to ageing of the sample and 
the electrolyte. Figure 4 shows a plot of the reference data recorded at $280 \mathrm{~K}$, normalised to the first data point at the beginning of the experiment. The degradation of $j_{\infty}(T=280 \mathrm{~K})$ can be described reasonably by an exponential function of time with an e-folding time of $\sim 27$ hours and a constant offset of 0.6 . Since every data point of the current measurement was recorded with a time stamp, we were able to drift-correct the raw data shown in Figure 3 by dividing each data point by the value of this exponential function at the respective time. The resulting drift-corrected data are plotted as circles in Figure 3. These data now show only a minimal scatter. Their error bars are larger than for the raw data because they include the uncertainty of the drift correction.

The error bar for the temperature is not shown in Figure 3 because it would be barely visible. The rated absolute accuracy of a Pt-100 sensor is better than $\pm 0.3 \mathrm{~K}$ within the investigated temperature range, measurement noise was typically of the order of $\pm 0.1 \mathrm{~K}$. Only for the data point at $333 \mathrm{~K}$, we state a temperature uncertainty of $\pm 1 \mathrm{~K}$ because of a thermal contact problem that occurred at this temperature. The size of the corresponding temperature error bar would be approximately the width of the symbol in this case.

\section{Discussion}

The drift-corrected data in Figure 3 can be reasonably well described by a single Arrhenius function with an activation energy of $0.46 \pm 0.1 \mathrm{eV}$ (blue dashed line). However, when comparing the data with the straight line of the Arrhenius fit, a slight curvature, particularly for the highest and lowest data points becomes apparent. In fact, the data are described even significantly better by a sum of two Arrhenius terms with activation energies of $E_{1}=0.86 \pm 0.07 \mathrm{eV}$ and $E_{2}=0.38 \pm 0.03 \mathrm{eV}$ (red continuous line). $E_{1}$ dominates towards higher temperatures, while $E_{2}$ describes the lower-temperature data. Because a curved line in an Arrhenius plot could also indicate a constant offset to an Arrhenius function, we also tested 
this fit (grey dotted line in Figure 3). Considering the substantial deviation at low temperatures, we can safely exclude this type of function.

We interpret the fact that $j_{\infty}(T)$ is best described by a sum of two Arrhenius terms with a higher activation energy for higher temperatures and a lower one for lower temperatures as an indication for two co-existing transport channels through the sample. The channel with the high activation energy $E_{l}$ has, for sufficiently high temperature, a much higher transport capacity. This can be interpreted such that this channel makes up the largest part of the sample area. The second transport channel comprises only a tiny fraction of the total current at elevated temperatures. Therefore, it only becomes visible at low temperatures because of its much smaller activation energy $E_{2}$. Accordingly, we assume that this channel makes up only a small fraction of the area of the sample exposed to the electrolyte. While we are convinced that the first channel corresponds to bulk grains of tungsten, we presently cannot definitely identify the smaller second channel. It has already been shown for, e.g., nickel that certain grain boundaries can feature a much faster diffusion of hydrogen than bulk material [15]. We speculate that it also may be easier for $\mathrm{H}$ to enter the $\mathrm{W}$ at a grain boundary intersecting the entry surface. In our sample, we have small, columnar grains. Accordingly, there is a high density of grain boundaries parallel to the permeation direction, which also intersect with the entry surface. For low temperatures, enhanced permeation along grain boundaries could dominate the total permeation current, while for higher temperatures permeation through the bulk of the grains would dominate because of the much larger effective area. We also cannot fully exclude that the channel with the low activation energy is due to small pinholes that go through the tungsten layer, but not through the supporting Pd foil. Unfortunately, such pinholes would be extremely difficult to detect because the sample would still be impermeable to light or any other indicator. What speaks against the existence of pinholes in the $\mathrm{W}$ film is that the activation energy $E_{2}=0.38 \pm 0.03 \mathrm{eV}$ of the second channel is still 
significantly higher than for the pure Pd support foil: here, literature values range around 0.14 eV (see, e.g., [16]). Furthermore, we measured the permeation current through a Pd foil without any $\mathrm{W}$ coating at $290 \mathrm{~K}$, with the same electrochemical potentials of $-350 \mathrm{mV}$ and $+400 \mathrm{mV}$ vs. SCE applied to the entry and exit sides of the foil. It was of the order of $200 \mu \mathrm{A} / \mathrm{cm}^{2}$. The permeation current through the W-coated Pd foil at $290 \mathrm{~K}$ was in all cases between about 50 and $80 \mathrm{nA} / \mathrm{cm}^{2}$. A leak current of $25 \mathrm{nA} / \mathrm{cm}^{2}$ (i.e., no more than half of the measured current) would already require a pinhole area of $12500 \mu \mathrm{m}^{2}$, which corresponds to a circular pinhole with a diameter of about $125 \mu \mathrm{m}$. Inspection of the sample with an optical microscope showed no defects of even nearly this size. Only with SEM small growth imperfections (typical size $\leq 5 \mu \mathrm{m}$ ) were found, but judging from a FIB cross-section, these do not appear to create open porosity in the $\mathrm{W}$ film.

The activation energy $E_{1}=0.86 \pm 0.07 \mathrm{eV}$ that we deduce from the high-temperature region of our data is still remarkably far below the value of $E_{\text {perm }}=1.43 \pm 0.26 \mathrm{eV} / \mathrm{H}$ derived from Frauenfelder's measurements at very high temperatures [3]. However, there is a gap of nearly $800 \mathrm{~K}$ between our highest temperature of $333 \mathrm{~K}$ and Frauenfelder's lowest temperature at $1100 \mathrm{~K}$. Looking at the trend of our data towards higher temperatures, the slope in the Arrhenius plot, and therefore the value of $E_{l}$, might increase further for higher temperatures than we were able to achieve in the experiments presented here. We therefore plan to investigate permeation of hydrogen isotopes through samples fabricated in the same way, i.e., a sputter-deposited tungsten layer supported by a Pd foil, in an experimental set-up designed for higher temperatures. Since the samples are very delicate, gas-phase loading appears risky. We therefore favour the option of ion-beam-driven permeation. Such experiments will show if the activation energy $E_{\text {perm }}$ indeed converges towards the high-temperature value, or if the particular fabrication method of our samples actually leads to a lower effective activation energy for $\mathrm{H}$ permeation than in bulk tungsten. 


\section{Summary}

We investigated the permeability of hydrogen through a $3.5 \mu \mathrm{m}$ thick magnetron-sputterdeposited PVD tungsten layer supported by a palladium foil. We used an electrochemical double cell similar to that described by Devanathan and Stachurski [8] filled with a highly viscous glycerol-phosphoric acid electrolyte mixture [9]. We applied potentiostatic charging with $-350 \mathrm{mV}$ vs. SCE at the entry side, and potentiostatic discharging at $+400 \mathrm{mV}$ vs. SCE ensuring a solute hydrogen concentration of zero at the exit side of the sample. By this, we measured the temperature dependence of the steady-state permeation current between 266 and $333 \mathrm{~K}$. We found that the measured data are described best by a sum of two Arrhenius terms with activation energies of $0.86 \pm 0.07 \mathrm{eV}$ and $0.38 \pm 0.03 \mathrm{eV}$. We speculate that if it is possible to extend the measurement to even higher temperatures, a gradual convergence of the data points at higher temperatures towards the value of $1.43 \pm 0.26 \mathrm{eV}$ measured by Frauenfelder at very high temperatures [3] may be observed. We plan to investigate this by using a sample produced by the same method as described here in an ion-beam-driven permeation set-up.

\section{Acknowledgement:}

This work has been carried out within the framework of the EUROfusion Consortium and has received funding from the European Union's Horizon 2020 research and innovation programme under grant agreement number 633053. The views and opinions expressed herein do not necessarily reflect those of the European Commission. 


\section{References}

[1] J. Völkl, G. Alefeld, Diffusion of Hydrogen in Metals, in: G. Alefeld, J. Völkl (eds.), Hydrogen in Metals I, pp.321-348, Springer Berlin Heidelberg, 1978 DOI: $10.1007 / 3540087052 \_51 \mathrm{~s}$

[2] J. Roth, K. Schmid, Phys. Scr. T145 (2011) 014031 DOI: $10.1088 / 0031-8989 / 2011 / \mathrm{T} 145 / 014031$

[3] R. Frauenfelder, J. Vac. Sci. Technol. 6 (1969) 388-397 DOI: $10.1116 / 1.1492699$

[4] T. Tanabe, Phys Scr. T159 (2014) 014044 DOI: 10.1088/0031-8948/2014/T159/014044

[5] P. Franzen et al., J. Nucl. Mater. 241-243 (1997) 1082-1086 DOI: 10.1016/S0022-3115(97)80198-5

[6] T. Ikeda, T. Otsuka, T. Tanabe, J. Nucl. Mater. 415 (2011) S684-S687 DOI: 10.1016/j.jnucmat.2010.12.007

[7] K.A. Moshkunov et al., J. Nucl. Mater. 404 (2010) 174-177 DOI: 10.1016/j.jnucmat.2010.07.011

[8] J.A.V. Devanathan, Z. Stachurski, Proc. R. Soc. Lond. A 270 (1962) 90-102 DOI: 10.1098/rspa.1962.0205

[9] R. Kirchheim, R.B. McLellan, J. Electrochem. Soc. 27 (1980) 2419-2425 DOI: $10.1149 / 1.2129486$

[10] A.J. Kumnick, H.H. Johnson, Metall. Trans. A 6 (1975) 1087-1091 DOI: $10.1007 / \mathrm{BF} 02661363$

[11] N. Boes, H. Züchner, J. Less Common Metals 49 (1976) 223-240 DOI: $10.1016 / 0022-5088(76) 90037-0$

[12] P. Manolatos et al., Corros. Sci. 37 (1995) 1797-1807 DOI: 10.1016/0010-938X(95)00081-T 
[13] P. Wang et al., Nucl. Instr. Meth. B 300 (2013) 54-61

DOI: 10.1016/j.nimb.2013.01.057

[14] P. Wang et al., Phys. Scr. T159 (2014) 014046

DOI: $10.1088 / 0031-8949 / 2014 / \mathrm{T} 159 / 014046$

[15] A. Oudriss et al., Scr. Mater. 66 (2012) 37-40

DOI: $10.1016 /$ scriptamat.2011.09.036

[16] E. Wicke, H. Brodowsky, H. Züchner, Hydrogen in Palladium and Palladium Alloys, in: G. Alefeld, J. Völkl (Eds.), Hydrogen in Metals II: Application-Oriented Properties, Springer-Verlag Berlin Heidelberg, 1978, pp. 73-155

DOI: 10.1007/3-540-08883-0_19 


\section{Figure captions}

Figure 1: Sketch of the electrochemical permeation set-up. The two individual cells are separated by the sample foil, which serves as the grounded common electrode of both cells.

Figure 2: SEM image of FIB cross-section through the permeation sample. The sample is tilted by $52^{\circ}$ relative to the surface normal. At the bottom of the image, a part of the $25 \mu \mathrm{m}$ thick Pd support foil is visible. The PVD-W layer on top of it has a thickness of $3.5 \pm 0.1 \mu \mathrm{m}$. The $50 \mathrm{~nm}$ thick Pd top layer is not visible here. An amorphous Pt:C layer was deposited prior to cross-sectioning to protect the sample surface during FIB milling.

Figure 3: Arrhenius plot of the temperature dependence of the steady-state permeation current $j_{\infty}(T)$. The open squares show the raw data. The circles show the data corrected for ageing effects. The data are best described by a sum of two Arrhenius terms (red continuous line). For comparison, fits with a simple Arrhenius term (blue dashed line) and an Arrhenius term with constant offset (gray dash-dotted line) are also shown.

Figure 4: Degradation of the steady-state permeation current $j_{\infty}(t)$ at $280 \mathrm{~K}$ after different times, normalised to the current $j_{o d}(t=0)$ at the beginning of the experiment. Ageing effects of the sample and the electrolyte over 8 days of measurement are approximated by an exponential function with a constant offset (continuous line), which was used for drift correction of the data in Figure 3. 


\section{Figures}

\section{Figure 1:}

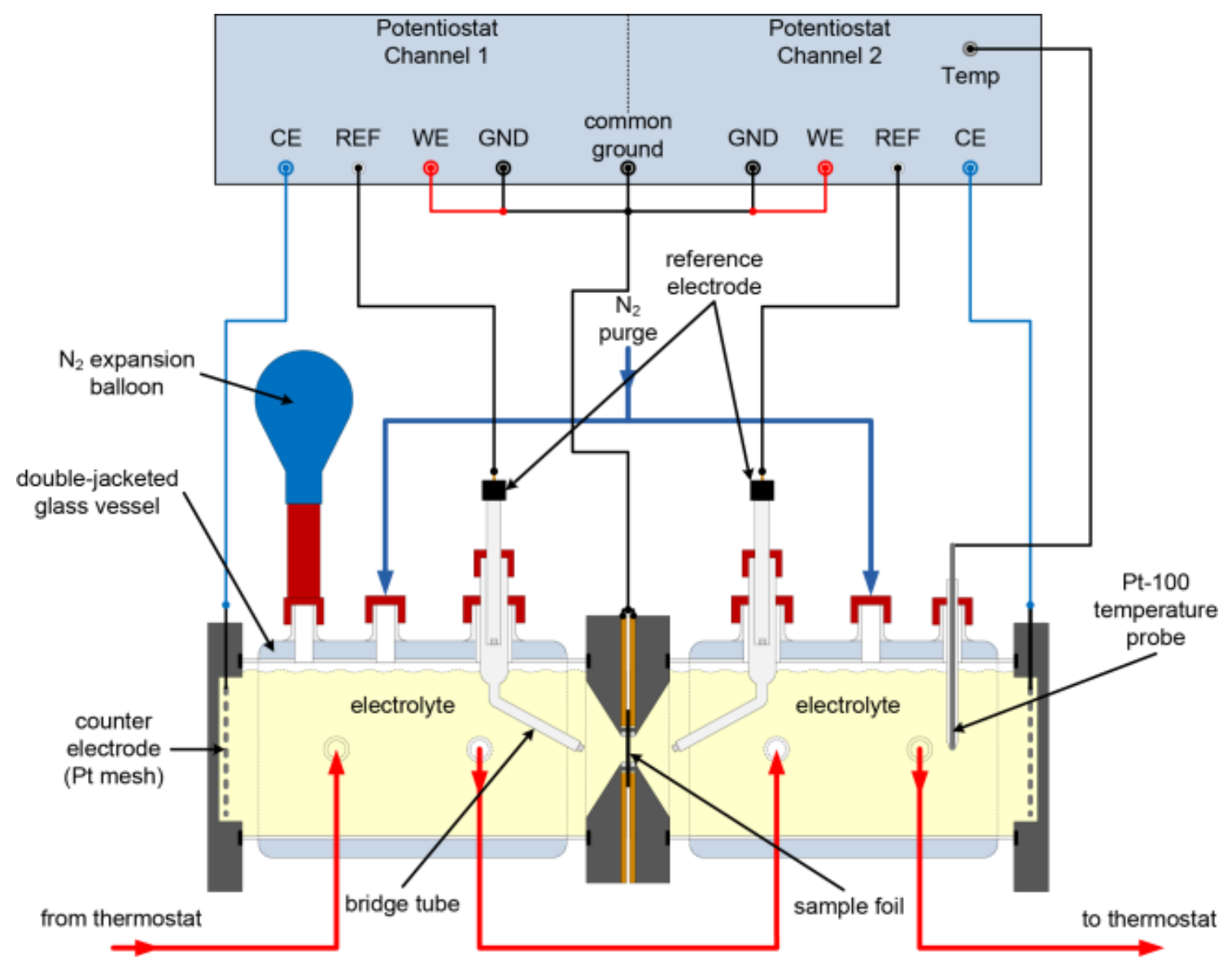

Sketch of the electrochemical permeation set-up. The two individual cells are separated by the sample foil, which serves as the grounded common electrode of both cells. 


\section{Figure 2:}

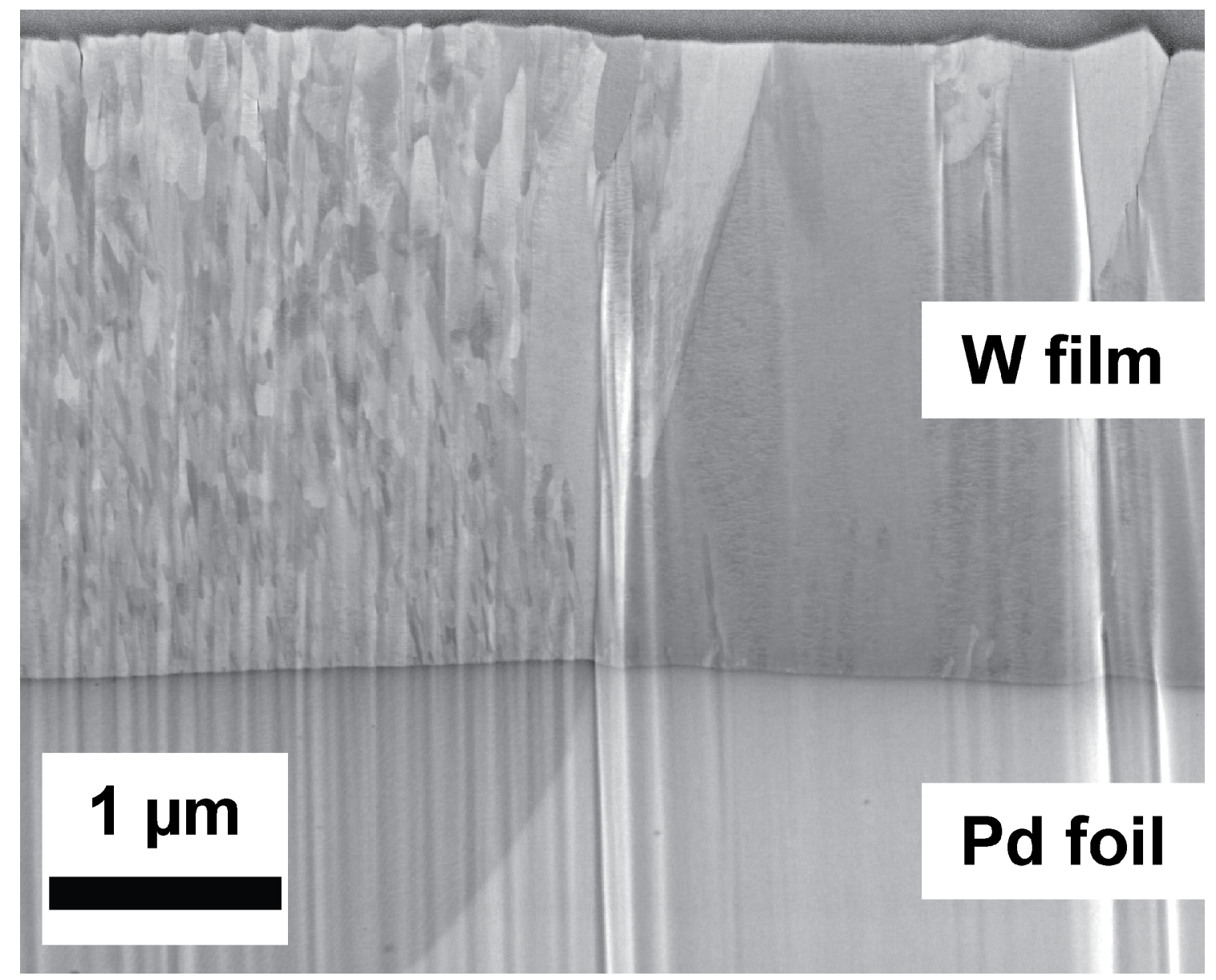

SEM image of FIB cross-section through the permeation sample. The sample is tilted by $52^{\circ}$ relative to the surface normal. At the bottom of the image, a part of the $25 \mu \mathrm{m}$ thick Pd support foil is visible. The PVD-W layer on top of it has a thickness of $3.5 \pm 0.1 \mu \mathrm{m}$. The 50 $n m$ thick Pd top layer is not visible here. An amorphous Pt:C layer was deposited prior to cross-sectioning to protect the sample surface during FIB milling. 


\section{Figure 3:}

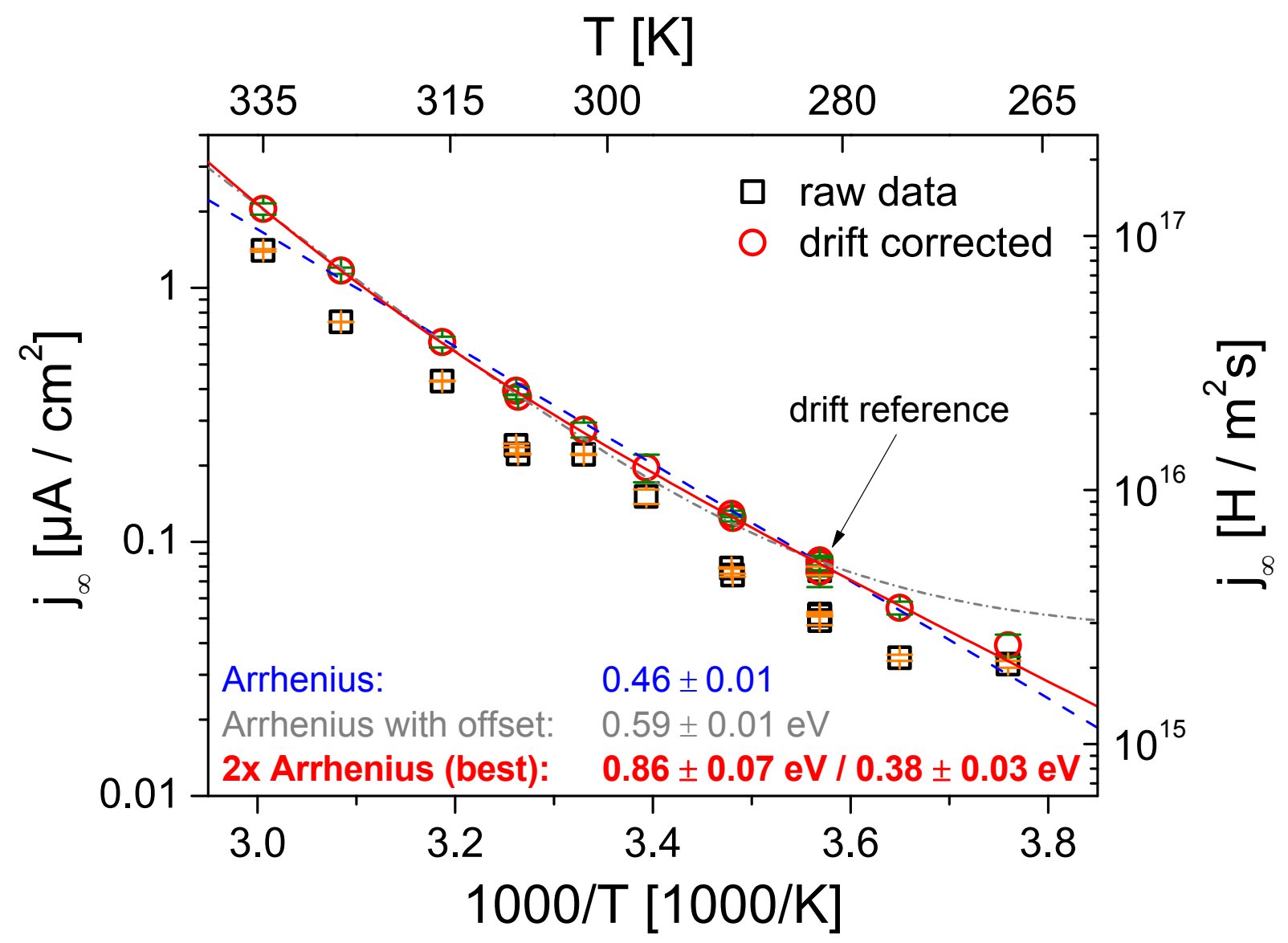

Arrhenius plot of the temperature dependence of the steady-state permeation current $j_{\infty}(T)$.

The open squares show the raw data. The circles show the data corrected for ageing effects.

The data are best described by a sum of two Arrhenius terms (red continuous line). For comparison, fits with a simple Arrhenius term (blue dashed line) and an Arrhenius term with constant offset (gray dash-dots) are also shown. 


\section{Figure 4:}

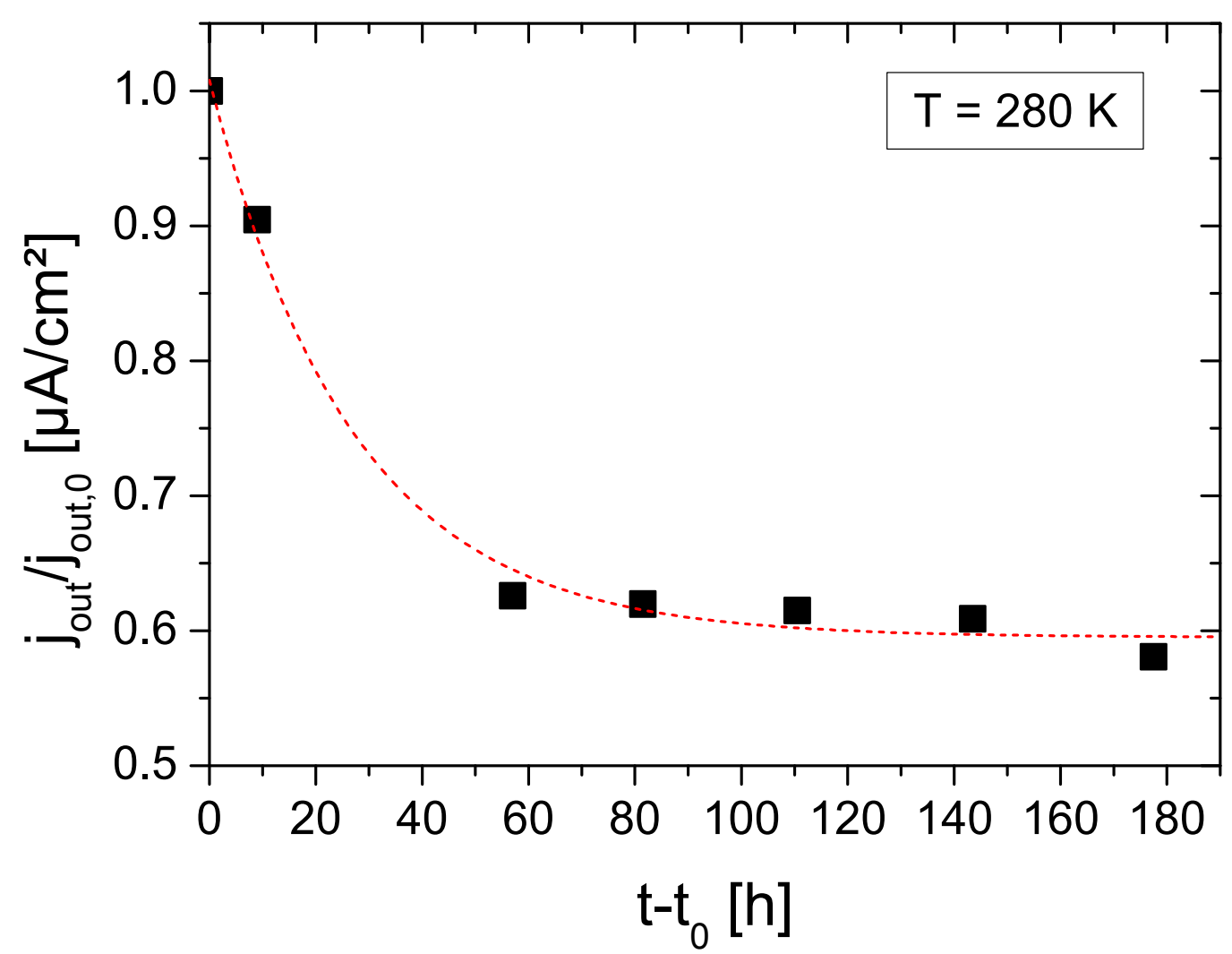

Degradation of the steady-state permeation current $j_{\infty}(t)$ at $280 \mathrm{~K}$ after different times, normalised to the current $j_{\infty}(t=0)$ at the beginning of the experiment. Ageing effects of the sample and the electrolyte over 8 days of measurement are approximated by an exponential function with a constant offset (continuous line), which was used for drift correction of the data in Figure 3. 